\title{
Self-evolution of Strange and Personalized Copperplate Etching Language
}

\author{
Zhu Liu \\ Guangxi Teachers Education University \\ Nanning, China
}

\begin{abstract}
With the particularity of copperplate etching, it requires workers to have high professional and technical quality. The technique factor has always been an organic part embedded in the art form of printmaking. To grasp the technique is the only way to study and master printmaking. Throughout the process of the development of copperplate etching, the new techniques are based on the experience of predecessors, long-term testing and improvement, and the existing techniques. And they can make better use of it. Then, it would provide a good platform for print-makers to express their personal ideas and feelings.
\end{abstract}

Keywords-copperplate etching language; personalized technique; strange

\section{INTRODUCTION}

People would start to learn the copperplate etching. And then, we are able to skillfully apply certain techniques to make creative writing. In the process, we would feel that people's energy is limited after all. If we use technologies in a piece of work, I am afraid that our energy should be used in dealing with techniques. To feel this, the author writes this article to start the discussion.

\section{The Purity of Personalized TeChNiQues}

"It is necessary to have effective tool s to do good work". The author thinks that the most basic thing an art explorer needs to do is to conquer his brush. If the art explorer can do this point, it is possible to do artistic creation. Many creators pay much attention to the different picture effects produced by different technologies and materials. However, they ignore the research on the richness of the same technology language. They only make the expansion of technology in the horizontal direction. And the author thinks that it is meaningful for the copperplate etching to make the development vertically. That is to say, we should make the research on the technology. Therefore, the purpose of technical simplification is to better express personal feelings and reflect the artistic nature of the work.

\section{A. Unique Visual Effects and Psychological Experience}

The copperplate etching often impresses with its heaviness. However, the feeling conveyed in my copperplate is different. It gives the impression of being light and steady, open, fresh and cozy. In the creation of the print "Forbidden Fruit Series", the artist uses unique techniques, and mainly takes subtle points as the elements. He makes the arrangement of different points. And then, with the arrangement of the language form, the artist would make the subjective creation. "Point, line and surface" become the most basic elements of drawing language. Leonardo da Vinci said: "The science of painting begins with a point firstly, followed by a line, again a surface, and finally a surface decides the body. Point is the most basic element. Painters often use "point" to activate the atmosphere of the picture, organize the structure of the object, and reflect an abstract life relation. For example, in Chinese history, there is an artist who uses "point" to express landscapes. And he was Mi $\mathrm{Fu}$ in the northern Song dynasty. He used the points in calligraphy to make the painting. And it would show different wind and rain changes of the natural mountains and rivers with different ink dots. People called it "the landscape of Mi". Another painter, Gong Xian followed Mi Fu's style. He made the form of "point" more abundant and full. The ever-changing landscapes of trees are attributed to the unified symbolic order. He put forward a rather constructive set of theories. The aim is to pursue the landscape that is both natural and close to subjective. And it would form unique, rich, natural style. And then, when the author created this series of "Forbidden Fruit", he used subjective desire to break down the objective and psychological feelings as well as my personal ideas into countless punctuated formal elements. And then, he reorganized and coordinated them. As De Chirico's points, "There is only what I can see with my eyes open, however, the better is still what I can see with my eyes closed". [1] Therefore, the unique visual effects of the picture produced by the techniques are corresponding to personal psychological feelings. Kandinsky once wrote: "From the point of extrinsic and intrinsic meaning, the point is the first element of the painting, especially for the print. In the special field of painting — the print, the point gives full play to the power of self-discipline. Tools of material ways show a wide variety of shape and sizes through this power. It provides all kinds of possibilities for a point to form different pictures. [2]

\section{B. Technical Limitations}

For all editions, they have their own limitations with taking the media of "print". And they are subject to certain restrictions on the arbitrariness and freedom of painting. All the visual effects of the copperplate etching must be obtained through the transformation of the medium of "print". And the 
characteristics of visual effects presented in the paintings often do not depend on the artist's will, but on the characteristics of the media of the print. In this paper, we could take the author's copperplate etching technique as the example. And it is derived from the practice of the author's print. To a certain extent, the creator would understand and make the use of new techniques. Each technique has its own limitations, which is also the characteristics and individuality. The author's hobbies, interests, personality and special feelings will make him prefer particular technique. And this technique limits certain themes. However, it would be applied to the performance of other themes. It is beneficial to express the emotion or create some artistic atmosphere. However, it is not suitable for other expressions of emotion or artistic atmosphere. Over time, it would form a unique style of the painter. However, the author believes that the "unique style" of the painters should be in a certain stage of the long artistic creation. And it will not be immutable. It won't be equivalent to personal style. Therefore, due to the uniqueness of individual techniques, it is suitable for representing non-deterministic objects such as softness, liquidity and non-realism. Then, the author created the series of print works like "Forbidden Fruit". As the author ever said, this series of Forbidden Fruit works are only in one stage of artistic creation. As the personal temperament changes, its techniques will also change accordingly.

\section{The Relationship between Inner Temperament and Personalized Technique}

The relationship between personal temperament or concerns and techniques has to go through a series of training of print creation. Finally, it will become part of their own feelings. However, the expressiveness of the technique should always be corresponding to the objective target expressed. In this way, the expression of the picture is linked with the technique by their own operational training. Then, it would form a relatively fixed style. Personal interests or concerns are subject to training in the process of using techniques. And it will gradually become an integral part of personal temperament as the techniques change.

In Shen Kuo's "Dream Stream Essays - Vol. 17", he saw Chinese landscape painting on the "ruined walls". In fact, it is influenced by his previous painting experience and traditional culture. "The benevolent see benevolence and the wise see wisdom". What you see objectively is similar. However, different viewers would make the adjustment of the objective things in accordance with their previous knowledge structure. When the author goes out to take a glimpse of the landscape, he will naturally think of the relevant styles showed in the copperplate etching or how to use his technique to express the ideas when he sees the woods or grasslands. Like Shen Kuo's "ruined walls", it refers to something that inspires and stimulates personal artistic accomplishment. And it would leave impression when people search for ink layer.

\section{THE COMPOSITION OF THE PICTURE AND EMOTIONAL EXPRESSION}

The painter Corot said: "I am careful in the pursuit and imitation of nature. I have never lose the opportunity to seize the moment touched my heart. Reality is part of art. Emotion is the whole thing of art. If it truly impresses the soul, our sincere feelings will convey to others." [3] And Rodin directly said: "Art is emotion." [4] The important psychological factor in artistic creation and artistic appreciation is emotion. All literature and art are the art of emotion. Without affection, there is no art. When artists carry out artistic creation, they first need to have the emotions. And then, the works of art can move others by the emotion. When their own artistic ideas and personal feelings change, the screen will also need to adapt to the medium of technology. It would accurately express personal thoughts and feelings. As a man who can make prints independently, the knowledge about the principle of the composition of the pictures has been digested. Also, it has become their subconscious. In the expression of personal ideas or emotions, it will be naturally mobilized from the brain to participate in the performance. During the creation of the series of copperplate etching "Blessing", creative ideas and emotions are no longer the performance of the previous stage of the "forbidden fruit" series. In the stage from the selection of creative theme to the sketch, they will consider to adopt which technique. When formal sketch is about to be put into practice as a work, it is natural to think of them - It would take one or several techniques as the medium to carry personal ideas. In this series of works, it tries to rely on the spirit of Buddhist thought to express the relationship between man and nature. The author joins the image of Buddha into copperplate etching. On the basis of the original techniques, it uses flying dust and line carving techniques to cooperate with it to increase the visual intensity of the picture. And then, it would achieve the artistic conception that I want to express. For example, after his visit to China, Mr. Tan Ping's prints have changed so much. And he changes his artistic concepts. He rationally chose art issue in the new cultural background. He was keen on the experience and expression of the picture texture. And planar space consciousness of the schemata remained the traditional closed style. The language of the copperplate etching "Miner" series finished in 1984 in China focused on the performance of the complicated feeling at the picture level. It enriched and clarified the natural texture of the copperplate etching with the technical means and visual experience. With the integration, it has conveyed unique connotation. Tan Ping ever said, in the year of 1987 and 1988, I consecutively created a series of copperplate etching "fish" works. Most of these works, it uses strong acid to corrode the edges of copperplate etching into incomplete shape. And it would strengthen the visual effects. Later, the images gradually disappeared. Then, it has derived and injected into the "line" language. The author stitched and connected the two pieces of broken copper with the intersecting lines. To use such expressions and actions was unprecedented in the print industry at that time. [5] After a short period of time, Tan Ping found his interest in the traditional principles and traditional paradigms of painting language weakened. In 1990, Tan Ping reopened his 
exploration of painting decisively. Yin Shuangxi said, from his print "miner" in university days and his recent "circle on blue and black background", we can see that he concentrates the development of Western art, which is from figurative to materialistic to purely abstract. In Tan Ping's art, it inherits the abstract heritage of mankind for thousands of years. [6] Therefore, with the constant changes of their artistic concepts and individuality propositions, it always affects the composition of the picture. At the same time, it incorporates different techniques.

\section{THE COORDINATION BETWEEN PERSONALIZED TECHNIQUES AND OTHER TECHNIQUES}

Due to the variety of techniques of copperplate etching, it is inevitable that various techniques and means will be used on the screen to achieve the desired effect. And these techniques would serve the theme and personal feelings. At the same time, we would encounter the issue of whether the techniques and techniques are coordinated. Unless, we use one technique in the creation, and there would have no such problems. According to the actual situation, we could coordinate new techniques with other techniques. And we should pay attention to two aspects through our long-term practice and thinking. The first is to understand and master the techniques you would use. And these techniques would present unique visual effects. And we can predict the combined effect of their own techniques to determine the general effect. On the other hand, I think the most important factor is that they should be consistent with your idea and content. Without thinking, you would piece together several techniques. It is certainly difficult to produce the desired effects. Taking my copperplate etching of "The Prayers Silent Night" as the example, three techniques are used in this copperplate etching. The first is unique individual techniques composed of delicate point elements. The second is the etching lines. The third is flying dust erosion. The first technique is characterized by its non-certainty of softness and fluidity, which are very fit for the performance of the memories in my paintings. The second technique and the third technique would give people the sense of heaviness and hierarchy and other effects. It provides the possibility for the volume, picture space, levels of black and white gray of Buddha. However, we are certain that any attempt to understand the techniques in his work in a comprehensive and systematic manner can cause disappointment and loss the interest. The painter Ernst wanted his images to keep his enigmatic and incomprehensible features. At the same time, it also made the viewers feel mesmerized. In Kong Guqiao's copperplate etching "Image - narrative and possibility", it takes seedpod of the lotus as the object of performance. First, he describes the lotus by using the technique of photographic plate making. Later, it makes the performance by using the ink-and-wash method and Mezzo tint successively. The method is to produce an uncertainty and different understanding of the negation of the objective original. And it expresses his unique ideas and propositions. Therefore, the use of technique in the picture actually embodies the inherent consistency of thought. And then, it achieves the agreement with the author, and conveys deep thoughts and the picture demands.

\section{CONCLUSION}

Each print artist will have different understanding and application of print language, which will inevitably produce different styles of prints. Throughout the history of the development of print, the content and form of the print are closely linked. Its form is the embodiment of concept, emotion and technology. Print language is actually an organism that includes skills, forms and contents. It is an organism formed by the self-discipline of print, the development of media materials and the professionalism of artists. Painters can express feelings with unique print language. Through the creation of the print series of "forbidden fruit" and "prayer for blessing", the selection and evolution of techniques, the continuous intervention of cultural information, the changes of views on things around, and the updating of personal conceptions have formed individual and unique print language.

\section{REFERENCES}

[1] Finkelstein. Zhang Aidong, Ku Zongbo, Wang Shengcai translation. Movie Screen in Surrealist Artistic Thought [M]. Nanjing: Jiangsu Fine Arts Publishing House. 2009

[2] Kandinsky. Luo Shiping, Wei Dahai, Xin Li translation [M]. Beijing: China Renmin University Press (CRUP), 2003

[3] He Zhengguang. Corot [M]. Shijiazhuang: Hebei Education Publishing House, 1998

[4] [France] Rodin. Fu Lei translation. Rodin Art Theory [M]. Tianjin Tianjin Social Sciences Press, 2006

[5] Tan Ping. Red Gate Gallery Exhibition [EB]. China Art Network, 2009-3-9

[6] Yin Shuangxi. Natural Abstraction - Interpretation of Tan Ping [EB] Tan Ping official website, 2010-7-11 